\title{
Mölders, Babette
}

\section{Mentoring zur Begleitung des Übergangs vom Studium in den Beruf - Konstruktionen eines Übergangs anhand einer Falldarstellung}

Schmidt-Lauff, Sabine [Hrsg.]; Felden, Heide von [Hrsg.]; Pätzold, Henning [Hrsg.]: Transitionen in der Erwachsenenbildung. Gesellschaftliche, institutionelle und individuelle Übergänge. Opladen ; Berlin ; Toronto : Verlag Barbara Budrich 2015, S. 239-251. - (Schriftenreihe der Sektion Erwachsenenbildung in der Deutschen Gesellschaft für Erziehungswissenschaft (DGfE))

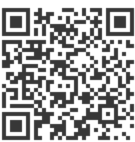

Quellenangabe/ Reference:

Mölders, Babette: Mentoring zur Begleitung des Übergangs vom Studium in den Beruf -

Konstruktionen eines Übergangs anhand einer Falldarstellung - In: Schmidt-Lauff, Sabine [Hrsg.];

Felden, Heide von [Hrsg.]; Pätzold, Henning [Hrsg.]: Transitionen in der Erwachsenenbildung.

Gesellschaftliche, institutionelle und individuelle Übergänge. Opladen ; Berlin ; Toronto : Verlag

Barbara Budrich 2015, S. 239-251 - URN: urn:nbn:de:0111-pedocs-130039 - DOI: 10.25656/01:13003

https://nbn-resolving.org/urn:nbn:de:0111-pedocs-130039

https://doi.org/10.25656/01:13003

in Kooperation mit / in cooperation with:

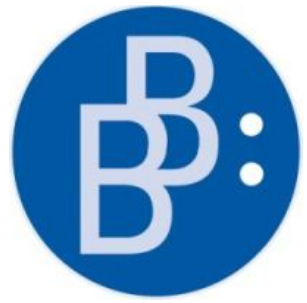

https://www.budrich.de

\section{Nutzungsbedingungen}

Dieses Dokument steht unter folgender Creative Commons-Lizenz: http://creativecommons.org/licenses/by-nc-nd/3.0/de/deed - Sie dürfen das Werk bzw. den Inhalt unter folgenden Bedingungen vervielfältigen, verbreiten und öffentlich zugänglich machen: Sie müssen den Namen des Autors/Rechteinhabers in der von ihm festgelegten Weise nennen. Dieses Werk bzw. dieser Inhalt darf nicht für kommerzielle Zwecke verwendet werden und es darf nicht bearbeitet, abgewandelt oder in anderer Weise verändert werden.

Mit der Verwendung dieses Dokuments erkennen Sie die Nutzungsbedingungen an.

\section{Terms of use}

This document is published under following Creative Commons-License: http://creativecommons.org/licenses/by-nc-nd/3.0/de/deed.en - You may copy, distribute and transmit, adapt or exhibit the work in the public as long as you attribute the work in the manner specified by the author or licensor. You are not allowed to make commercial use of the work or its contents. You are not allowed to alter, transform, or change this work in any other way.

By using this particular document, you accept the above-stated conditions of use.

\section{Kontakt / Contact:}

\section{peDOCs}

DIPF | Leibniz-Institut für Bildungsforschung und Bildungsinformation Informationszentrum (IZ) Bildung

E-Mail: pedocs@dipf.de

Internet: www.pedocs.de

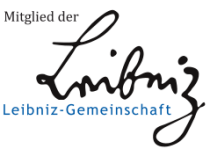




\section{Transitionen in}

\section{der Erwachsenenbildung}

Gesellschaftliche, institutionelle und individuelle Übergänge

Sabine Schmidt-Lauff

Heide von Felden

Henning Pätzold (Hrsg.)

Schriftenreihe der Sektion

Erwachsenenbildung

der Deutschen Gesellschaft für Erziehungswissenschaft

\section{DGfE}


Schriftenreihe der Sektion

Erwachsenenbildung

in der Deutschen Gesellschaft für

Erziehungswissenschaft (DGfE) 
Sabine Schmidt-Lauff

Heide von Felden

Henning Pätzold (Hrsg.)

\section{Transitionen in \\ der Erwachsenenbildung}

Gesellschaftliche, institutionelle und individuelle Übergänge

Verlag Barbara Budrich

Opladen • Berlin • Toronto 2015 
Bibliografische Information der Deutschen Nationalbibliothek

Die Deutsche Nationalbibliothek verzeichnet diese Publikation in der Deutschen Nationalbibliografie; detaillierte bibliografische Daten sind im Internet über

http://dnb.d-nb.de abrufbar.

(C) 2015 Dieses Werk ist bei Verlag Barbara Budrich erschienen und steht unter folgender Creative Commons Lizenz: http://creativecommons.org/licenses/by-nc$\mathrm{nd} / 3.0 / \mathrm{de} /$

Verbreitung, Speicherung und Vervielfältigung erlaubt, kommerzielle Nutzung und Veränderung nur mit Genehmigung des Verlags Barbara Budrich.

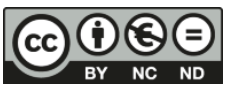

Dieses Buch steht im OpenAccess Bereich der Verlagsseite zum kostenlosen Download bereit ( https://doi.org/10.3224/84740753)

Eine kostenpflichtige Druckversion kann über den Verlag bezogen werden. Die Seitenzahlen in der Druck- und Onlineversion sind identisch.

\section{ISBN 978-3-8474-0753-9 \\ eISBN 978-3-8474-0915-1 \\ DOI $\quad 10.3224 / 84740753$}

Umschlaggestaltung: Bettina Lehfeldt, Kleinmachnow - www.lehfeldtgraphic.de

Lektorat und Satz: Judith Henning, Hamburg - www.buchfinken.com

Verlag Barbara Budrich, Opladen, Berlin \& Toronto

http://www.shop.budrich-academic.de/ 


\section{Inhalt}

\section{Vorwort}

Heide von Felden/Sabine Schmidt-Lauff

Transitionen in der Erwachsenenbildung: Übergänge im gesellschaftlichen Wandel, im Fokus von Forschung und aus Sicht pädagogischer Professionalität.

Key Notes

Ortfried Schäffter

Übergangszeiten - ,Transitionen ' und ,Life Trajectories'. Navigieren durch Bildungslandschaften im Lebensverlauf....

Andreas Walther

Übergänge im Lebenslauf: Erziehungswissenschaftliche Heuristik oder pädagogische Gestaltungsaufgabe?

Theoretische Perspektiven

Ulla Klingovsky/Susanne Pawlewicz

Übergang, Unsicherheit und Unterbrechung: Scheitern als Chance zur

Differenzbildung.....

Heide von Felden

Lernwelten und Transitionen: Übergangsforschung als

Lernweltforschung

Nicole Hoffmann

Übergangsstrukturen im Feld der pädagogischen Beratung

Erwachsener - ethnografische Perspektiven im Anschluss an Arnold

van Gennep 
Claudia Lobe

Biografieorientierte Transitionsforschung als Teilnehmerforschung Wie sich Erwachsenenbildungsteilnahme als biografische Transition untersuchen lässt.

Berufliche Übergänge

Melanie Benz-Gydat

Der Berufseinstieg als Transition: Vom Studium in die andragogische

Praxis

Barbara Lindemann

Die Relevanz von sozialen Kontakten beim Übergang vom Studium in das Erwerbsleben. Ergebnisse aus einer Befragung der Münchner Magisterpädagoginnen und Magisterpädagogen....

Jörg Schwarz/Franziska Teichmann/Susanne Maria Weber

Transitionen und Trajektorien.

Bettina Ülpenich

Der Weg in die Schule - Passagenbewältigung von

Lehramtsanwärtern und -anwärterinnen in Eigenkonstruktion

Beatrix Niemeyer-Jensen/Merle Hinrichsen

Möglichkeitsräume (re)konstruieren - Biographische

Aneignungsprozesse zwischen Schule und Erwerbstätigkeit

Monique Landberg/Peter Noack

Prädiktoren von berufsbezogenen Orts- und Richtungswechseln von jungen Erwachsenen und die Rolle von Agency.

Johanna Gebrande/Rudolf Tippelt

Basiskompetenzen am Übergang in die Nacherwerbsphase

Marion Fleige

Nutzenvorstellungen von Weiterbildungsteilnehmenden in beruflichen und erwerbsbiographischen Übergangssituationen 
Yeşim Kasap Çetingök

Die transitionengerechte Konzeptualisierung der psychosozialen

Beratung für die Erwachsenen und Ermöglichung von

Bildungsprozessen

Stephanie Günther/Joachim Ludwig

Transformationen pädagogischen Wissens bei nebenberuflichen

Kursleitenden im Kontext pädagogischer Weiterbildung

Babette Mölders

Mentoring zur Begleitung des Übergangs vom Studium in den Beruf -

Konstruktionen eines Übergangs anhand einer Falldarstellung

Barbara Nienkemper

Abschlussorientierte Tests und Prüfungen im Kontext von

individuellen Übergängen bei funktionalem Analphabetismus

\section{Institutionelle und organisationale Übergänge ............. 265}

Matthias Alke

Institutionelle Übergänge durch interorganisationale Kooperationen.

Eine empirische Rekonstruktion institutioneller Wandlungsprozesse

von Weiterbildungsorganisationen

Henning Pätzold

Organisationale Übergänge zwischen Weiterbildung und Wirtschaft

Alf-Tomas Epstein

Interessegenese, Weitergabe von Verbandserbe und Förderprozesse in

Jugendverbänden als Beiträge zur Übergangsgestaltung ....

Helmut Bremer/Mark Kleemann-Göhring

Jugendverbände als Bildungsorte im „Feld des Übergangs“ .303

Inga Truschkat/Luisa Peters

Die Transfergesellschaft als personen(un)bezogene Dienstleistung am Übergang von Arbeit in Arbeit 
Inhalt

Steffi Robak/Claudia Pohlmann/Lena Heidemann

Anschlusslernen und Lern-Verwertungsinteressen am Beispiel von

Bildungsurlaub

Christina Salland/Melanie Franz/Timm C. Feld

Zur Gestaltung von Übergängen im Kontext wissenschaftlicher

Weiterbildung - Rolle von und Herausforderungen für Universitäten

Autorenangaben

359 


\section{Mentoring zur Begleitung des Übergangs vom Studium in den Beruf - Konstruktionen eines Übergangs anhand einer Falldarstellung}

\section{Einleitung}

Der folgende Aufsatz beschäftigt sich mit den möglichen Unterstützungsfunktionen von Mentoring zur Begleitung von Hochschulabsolventinnen ${ }^{1}$ während des Übergangs von der Hochschule in den Beruf. Basis der Ausführungen ist das laufende Dissertationsprojekt „Mentoring zur Begleitung von Übergängen“, das mithilfe biographisch-narrativer Interviews mit ehemaligen Mentees verschiedener Mentoringprogramme der Frage nachgeht, welche Funktionen Mentoring für Hochschulabsolventinnen in der Begleitung und zur Bewältigung der tendenziell risikoreichen und individualisierten Übergangspassage umfassen kann. Übergangsprozesse werden im vorliegenden Projekt im Anschluss an Welzer (1993) als Transitionen gefasst und in ihrem biographischen Kontext und Sinnzusammenhang betrachtet. Mentoring kann hierbei als eine individuelle Begleitung des Bildungs- und Lernprozesses im Übergang verstanden werden. Anhand einer Falldarstellung werden im Folgenden die Konstruktionen eines Übergangs als ein Zwischenergebnis des Dissertationsprojekts präsentiert. Bevor das Projekt und dessen methodische Anlage vorgestellt werden, soll zunächst das Konzept Mentoring als eine Form von (Bildungs-)Beratung fokussiert werden.

\section{Mentoring}

Klassischerweise wird als Bezug und Ursprung des Mentoring-Konzepts die griechische Mythologie herangezogen: ${ }^{2}$ In der Odyssee, einem Epos von Homer, in dem die Abenteuer des Odysseus beschrieben werden, lässt Odysseus seinen Sohn Telemachos während seiner zehnjährigen Irrfahrt von seinem Freund und Vertrauten Mentor erziehen, beraten und leiten. Mentoring lässt sich der Sage zufolge als eine Beratungs- und Unterstützungsbeziehung zwischen einer erfahrenen Person (Mentor/-in) und einer weniger erfahrenen Person (Mentee) beschreiben. Nele Haasen (2001) definiert Mentoring als „eine Eins-zu-Eins-Beziehung zwischen einem Berater oder einer Beraterin

1 Es wurden ausschließlich weibliche Mentees befragt (s. Punkt 3: Methodisches Vorgehen).

2 Vgl. zum folgenden Abschnitt auch Berkels (2014). 
und einem/einer Ratsuchenden. Beide führen über einen längeren Zeitraum regelmäßig Gespräche. Darin können Fragen aus dem Alltag ebenso besprochen werden wie allgemeinere Themen. Das Ziel ist die Weiterentwicklung der Persönlichkeit und der Fähigkeiten des oder der Mentee und die Förderung seiner oder ihrer beruflichen Karriere" (Haasen 2001, S. 15). Mentoring wird demnach als eine Form von Beratung verstanden, die verschiedene Ziele verfolgen kann und eine längerfristige Prozessbegleitung darstellt. Im Kontext von Bildungsberatung werden insbesondere Orientierungs- und Entscheidungsprozesse in Bezug auf die Gestaltung der Berufs- und Bildungsbiographie fokussiert, es geht um den Entwurf von (berufs- und bildungs-)biographischen Planungsschritten unter Nutzung prinzipiell vorhandener Ressourcen der Ratsuchenden (vgl. Schlüter 2010). Als eine (eher informelle bzw. halb-formalisierte Form $^{3}$ ) von Bildungsberatung kann Mentoring in unterschiedlichen Lebensphasen und Übergangsprozessen bzw. Umbruchsituationen eine begleitende und beratende Funktion erfüllen. Dabei kann es etwa um eine Vorbereitung auf die Übernahme neuer (Führungs-)Aufgaben gehen, um die Begleitung bei der Einmündung in ein neues Unternehmen oder auch die erstmalige Berufseinmündung. Mentoren und Mentorinnen können in beratender Weise an diesen Schwellen stattfindende Lern- und Bildungsprozesse unterstützen und begleiten, die durch die Konfrontation der Mentees mit neuen Situationen und Handlungsanforderungen angeregt werden.

Mentoringbeziehungen lassen sich nach dem Grad ihrer Formalisierung unterscheiden. Zum einen gibt es informelle, durch Initiative von Mentee und/oder Mentor/-in entstandene Beziehungen, wie es in der griechischen Sage zum Ausdruck kommen. Seit den 1990er Jahren lässt sich in Deutschland zudem eine Formalisierung dieser Beziehungen durch die Etablierung von Mentoringprogrammen in den verschiedensten beruflichen Handlungsfeldern von Hochschulen über Unternehmen bis hin zu Verwaltungen und Non-Profit-Organisationen konstatieren. Kennzeichen dieser formalen Programme ist, dass Mentee und Mentor/-in durch eine Programmorganisation in einem Matchingprozess zusammengeführt werden. Darüber hinaus umfassen sie eine Vorbereitung der Tandempartner/-innen auf das Mentoring, ihre

3 In Bezug auf die Differenzierung von Beratung in informelle, halb-formalisierte und stark formalisierte Beratung (Sickendiek/Engel/Nestmann 2008, S. 23) lässt sich Mentoring zwischen informeller Beratung - als alltägliche Beratung unter Verwandten, Bekannten, Kollegen und Kolleginnen - und halb-formalisierter Beratung verorten. Halb-formalisierte Beratung wird durch Angehörige unterschiedlicher, oftmals (sozial-)pädagogischer oder psychosozialer Berufe als integraler Bestandteil ihres professionellen Handelns erbracht, nicht jedoch als abgegrenzte oder vorrangige Aufgabe bzw. Tätigkeit wie es bei professionellen Beratern und Beraterinnen mit entsprechender Qualifikation und Beratungskompetenz der Fall ist (vgl. auch Lorenzen 2014, S. 141 ff.). Von dieser Systematisierung zu unterscheiden ist die Abgrenzung von informellem und formalem Mentoring (s.u.). 
Rollen und damit verbundene Aufgaben sowie eine Begleitung der Tandems. Die Mentoringbeziehung wird klassischerweise ergänzt und gerahmt durch Auftakt-, Zwischenbilanzierungs- und Abschlussveranstaltungen, ein Workshopprogramm für Mentees und/oder Mentoren und Mentorinnen sowie Networking-Bausteine. Um am Programm teilnehmen zu können, durchlaufen Mentees ein Bewerbungs- bzw. Auswahlverfahren.

\section{Methodisches Vorgehen}

\subsection{Forschungsinteresse und Fragestellung}

Das Forschungsinteresse des Dissertationsprojekts, aus dem die im Rahmen des Aufsatzes analysierte Falldarstellung stammt, richtet sich auf die Funktionen, die Mentoring im Übergang vom Studium in den Beruf entfalten kann. Konkret untersucht es die Frage, welchen Einfluss Mentoring zur Begleitung der Übergangspassage vom Studium in den Beruf besitzen kann. Dabei wird eine biographietheoretische Perspektive eingenommen und es werden individuelle Bewältigungs-, Deutungs- und Handlungsmuster der Übergängerinnen rekonstruiert, die Einsicht in Transitionsprozesse und deren Konstruktion geben.

Die methodische Umsetzung erfolgt mithilfe biographisch-narrativer Interviews ${ }^{4}$ die nach Fritz Schütze (1983) analysiert werden. Narrative Interviews erlauben es, die lebensgeschichtlichen Erfahrungen in der Erzählung mitzuteilen. Im Rahmen der Auswertung lässt sich eine Rekonstruktion und Interpretation der Erfahrungen vornehmen. Damit lassen sich die Konstruktion der Zusammenhangs- und Sinnbildung biographischer Erfahrungen sowie die biographische Eigenlogik von Lernprozessen im Mentoring herausarbeiten - d.h., es können individuelle Erfahrungen und gleichermaßen die sie rahmenden gesellschaftlichen Strukturen und Bedingungen fokussiert werden (vgl. auch Berkels 2014). Ein Blick auf die Gesamtbiographie ist für die vorliegende Studie notwendig, denn ,,[g]eht es darum, etwas über Antizipationen und Erfahrungen in einer Übergangssituation von der Hochschule in den Beruf zu erfahren, gehören nicht nur die aktuellen Erfahrungen der Betroffenen in den Untersuchungskontext, sondern auch die biographischen und speziell die bildungsbiographischen, auf deren Basis sie sich ja erst konturieren" (Welzer 1990, S. 16). Vorausgegangene Erfahrungen der Individuen, insbesondere mit Übergängen, und daraus gebildete Deutungs- und Handlungsmuster beeinflussen das Erleben eines Übergangs sowie die darauf basierenden Orientierungs- und Bewältigungsversuche.

4 Die Interviewpartnerinnen wurden im Sinne eines möglichst offenen Erzählimpulses gebeten, ihre Lebensgeschichte zu erzählen. 
Infolge von Prozessen des strukturellen Wandels sind Lebensverläufe zunehmend als deinstitutionalisiert und entstandardisiert zu kennzeichnen. Übergänge in der (fortgeschrittenen oder auch zweiten, reflexiven) Moderne verlaufen gemäß soziologischen Gesellschaftsanalysen (bspw. Beck 1986, Beck u.a. 1996) individualisierter, differenzierter, komplexer und auch risikoreicher als in (vor-)modernen Zeiten, Übergangsphasen verlängern sich. Es lässt sich daher von einer „Biographisierung“ des Übergangs sprechen (Oehme 2007, S. 60 ff.), d.h., Übergänge bringen Anforderungen an ihre biographische Gestaltung mit sich. Demnach stellen sich Bildungs- und Lernprozesse als Anforderung sowie zugleich als Bewältigungsressource im Übergang dar (vgl. Pohl/Stauber/Walther 2006, S. 186), Mentoring kann in diesem Kontext als Prozessbegleitung fungieren.

Das Transitionskonzept nach Welzer ist zur Konzeptualisierung von Übergängen für die vorliegende Studie aus verschiedenen Gründen besonders passfähig: Zum einen werden Transitionen von Welzer als soziale Prozesse gefasst, an denen neben dem Individuum im Übergang weitere Personen beteiligt sind und ,,in denen Gewohnheiten, Handlungs- und Deutungsroutinen sowie Wissensbestände aller Beteiligten Modifikationen unterworfen sein können“ (Welzer 1993, S. 37). Im vorliegenden Projekt stellt sich die Frage nach der Rolle von Mentoring in diesen sozialen Prozessen.

Zudem sind Ausgangs- und Ankunftspunkte von Transitionen nach Welzer (1993) nicht präzise definierbar. In Bezug auf den Übergang vom Studium in den Beruf lässt sich fragen, wann eine Transition beginnt: nach Erhalt des Abschlusszeugnisses, vielleicht auch Monate früher, wenn dem/der Übergänger/-in bewusst wird, dass das Studienende bevorsteht? Ebenso lässt sich das Ende der Transition nicht eindeutig bestimmen: Ist die Transition mit der Unterschrift des Arbeitsvertrages abgeschlossen oder erst einige Zeit später, nachdem das Individuum im Berufsleben angekommen ist?

Transitionen lassen sich darüber hinaus in Bezug auf ihre Vorhersagbarkeit und Freiwilligkeit differenzieren (Welzer 1990, S. 43), die sich als Merkmale auf die Bewältigungsanforderungen, die Bedeutung bzw. die Qualität der Übergangserfahrung auswirken. Hier findet sich der Bezug zu Haltungen der Individuen gegenüber Transitionserfahrungen, d.h. den von Schütze als „Prozessstrukturen des Lebenslaufs“"5 (1983 S. 283) bezeichneten Haltungen der Biographieträger/-innen zu ihren Erfahrungen. In einer Ver-

5 Die von Schütze herausgearbeiteten Prozessstrukturen sind biographische Handlungsschemata, die intentional, von dem/der Biographieträger/-in geplant sind. Institutionelle Ablaufmuster der Lebensgeschichte kennzeichnen sich durch eine Orientierung an gesellschaftlichen oder organisatorischen Erwartungsfahrplänen. In Verlaufskurvenprozessen wird das Individuum durch lebensgeschichtliche Ereignisse überwältigt und kann nur noch ,konditionell' reagieren. Wandlungsprozesse stellen eine Erweiterung der Erlebnis- und Handlungsmöglichkeiten der Biographieträger/-innen dar, die sich überraschend entfalten (vgl. Schütze 1983, S. 283). 
bindung von Transitionen und Prozessstrukturen lässt sich fragen: Welche Haltungen entwickeln Biographieträger/-innen zu Transitionen? Wie erleben sie sich selbst? Welche Folgen hat dies für Mentoring zur Begleitung der Transition?

\subsection{Charakterisierung der von den Befragten durchlaufenen Mentoringprogramme}

Die befragten Mentees haben an verschiedenen Mentoringprogrammen teilgenommen: Dazu gehören zwei Mentoringprogramme für Studierende bzw. Absolventen und Absolventinnen aller Fachrichtungen im Übergang Hochschule-Beruf, angeboten durch Universitäten in NRW, sowie das Programm eines Begabtenförderwerkes, dessen Fokus insbesondere auf Führungskräfteentwicklung liegt. Alle Programme verfolgen das Ziel, die Phase des Übergangs zu begleiten, d.h. etwa die ersten Schritte des Karrierewegs und eine frühzeitige Karriereplanung zu unterstützen und damit langfristig zu einer Erhöhung des Anteils von Frauen in Führungspositionen beizutragen. ${ }^{6}$

\section{$4 \quad$ Stand der Forschung}

In Deutschland fokussiert die empirische Forschung vor allem formale Mentoringprogramme, die zumeist der Frauenförderung dienen. Dabei handelt es sich fast ausschließlich um wissenschaftliche Begleitforschung zu bzw. Evaluation von Mentoringprogrammen an Hochschulen bzw. an der Schnittstelle Hochschule-Beruf oder Schule-Hochschule. Vereinzelt werden auch Programme aus der Wirtschaft analysiert, die der Förderung des (weiblichen) Führungsnachwuchses dienen. Im Kontext von Mentoring in Wirtschaftsunternehmen werden Programme zudem als Instrument der Personalentwicklung betrachtet sowie deren Wirkungen auf die Organisationsentwicklung erforscht. Auch der Einsatz von Mentoring im Rahmen betrieblicher Gleichstellungspolitik wird fokussiert.

Aktuell liegen in Bezug auf die langfristigen Wirkungen von Mentoringprogrammen auf die individuellen Berufs- und Karriereverläufe von Mentees kaum Studien vor. Es existiert nur eine geringe Anzahl von Langzeiterhebungen oder Nachbefragungen von Mentees zurückliegender Mentoringjahrgänge (vgl. Kurmeyer 2012, S. 12). In erster Linie liegen Evaluationen vor,

6 Im Zeitverlauf gab es bei den universitären Programmen Linien, die sich auch an männliche Mentees richteten. Von diesen (wenigen) männlichen Mentees hat sich niemand zur Teilnahme an der Untersuchung bereitgefunden, daher wurden ausschließlich weibliche Mentees befragt. Das Programm des Begabtenförderwerkes richtet sich ausschließlich an weibliche Mentees. 
die sich direkt an die Programmteilnahme anschließen und daher vor allem Aussagen über die Güte der durchgeführten Programme ermöglichen sollen.

Die Betrachtung und Untersuchung von Mentoringprozessen in biographischer Perspektive geschieht bislang nur in Einzelfällen (vgl. Schell-Kiehl 2007; Kurmeyer 2012). Eine Verbindung der Forschung zu Mentoring mit Übergangsforschung und speziell an der Schwelle Hochschule-Beruf ist aktuell noch nicht erfolgt. Die vorliegende Forschungsarbeit soll diese Verbindung einer biographieanalytischen Untersuchung von Mentoringprozessen mit Übergangsforschung herstellen.

\section{Falldarstellung Frau Winkler}

\subsection{Biographische Kurzbeschreibung}

Frau Winkler wird 1976 in Bayern geboren und ist das mittlere Kind von drei Geschwistern: Sie hat eine jüngere Schwester und einen älteren Bruder. Ihr Vater ist Arzt, ihre Mutter Medizinisch-technische Assistentin. Als Frau Winkler fünf Jahre alt ist, gehen ihre Eltern mit ihr und den Geschwistern für sieben Jahre nach Saudi-Arabien, um dort in der Entwicklungszusammenarbeit tätig zu sein. In dieser Zeit besucht Frau Winkler eine internationale Schule. Die Familie wohnt zusammen mit anderen Entwicklungshelfern und -helferinnen in einer Siedlung in der Wüste und hilft dort, ein Krankenhaus aufzubauen. Nachdem der Vertrag ihres Vaters nicht verlängert wird, kehrt die Familie nach Deutschland zurück und lebt wieder in Bayern, wo Frau Winkler ein Gymnasium besucht.

Etwa 1992 trennen sich die Eltern, wodurch eine schwierige familiäre Situation entsteht. Nach ihrem Abitur 1994 absolviert Frau Winkler ein freiwilliges soziales Jahr als Volunteer in einem Obdachlosenheim in einer nordenglischen Großstadt. Sie ist mit ihrer Arbeit dort jedoch völlig überfordert. Belastet durch die weiterhin schwierige familiäre Situation entschließt sie sich nach acht Monaten vorzeitig abzureisen. 1996 beginnt sie ein Magisterstudium der Fächer Politik und Anglistik, mit dem Ziel, Journalistin zu werden. Sie wechselt von Anglistik zur Ethnologie, für die sie durch ihre Auslandserfahrungen ein großes Interesse hegt. Frau Winkler arbeitet von 2000 bis 2008 neben ihrem Studium bei einem großen Rundfunksender. Zum Ende ihres Magisterstudiums entdeckt sie ihr Interesse an Medizin-Ethnologie. Nach dem schnellen und guten Abschluss des Ethnologiestudiums entscheidet sie sich Medizin zu studieren. Sie nimmt das Studium 2002 auf, zeitgleich erhält sie das Angebot für ein Volontariat bei dem Rundfunksender, für den sie im Nebenjob tätig ist. Sie denkt intensiv über die Annahme der begehrten Volontariatsstelle nach, entschließt sich jedoch gegen einen journalistischen Berufsweg und studiert weiter Medizin. Von 2004 bis 2005 nimmt sie am 
Mentoringprogramm ihrer Universität teil, um einen Einblick in ein mögliches berufliches Tätigkeitsfeld zu erhalten. Zur Finanzierung ihrer Auslandsaufenthalte während des Studiums bewirbt sie sich um verschiedene Stipendien, die sie auch erhält. Frau Winkler absolviert u.a. von 2005 bis 2006 einen Forschungsaufenthalt in Südostasien, wo sie eine Erhebung für ihre Doktorarbeit im Bereich Frauenheilkunde durchführt. Sie geht in das praktische Jahr als Ärztin, 2008 macht sie ihr Examen. Von 2009 bis 2011 ist sie klinisch tätig. Sie stellt ihre Doktorarbeit 2010 fertig und promoviert im selben Jahr. Weil sie die wissenschaftliche Arbeit vermisst, stellt sie einen Antrag bei einer großen Forschungs- und Wissenschaftsförderungseinrichtung, um sich selbst eine Stelle zu schaffen. Der Antrag wird bewilligt und sie unterbricht ihre Facharztausbildung, um Mitte 2011 ihre Stelle als wissenschaftliche Mitarbeiterin zu beginnen. Für die Zukunft plant sie ihre Facharztausbildung zu beenden, beschäftigt sich jedoch zunächst mit dem Abschluss ihres Projekts.

\subsection{Thema des Interviews}

Das Thema des Interviews ist Frau Winklers Suche nach sich selbst. Darauf bezogen werden Heimatlosigkeit und Fremdheit von Frau Winkler fokussiert, als Gefühle, die sie über ihre Biographie hinweg begleiten und besonders an biographischen Übergängen deutlich werden. Dies lässt sich exemplarisch bei der Rückkehr von Saudi-Arabien nach Bayern feststellen:

das war ungefähr so vier fünf Monate, das Ankommen war wirklich richtig schlimm [...] also das war so dieser Übergang so, wir gehören nicht dazu, ist bei mir so hängen geblieben "7 (Z. 545-545). Weiter evaluiert Frau Winkler: „dieses Gefühl von ich gehör nicht richtig dazu oder meine Erfahrungen sind andere als die von anderen Kindern und ich hab nicht so eine richtige Heimat, das ist was, was eigentlich bis heute (5) also was mich bis heute begleitet (Z. 564-569).

Frau Winkler stellt im Interview einen Entwicklungsprozess dar, der oftmals von außen angestoßen wird und den Beginn einer Verlaufskurve einläutet. Durch die Entwicklung biographischer Handlungsschemata gelingt es ihr jedoch, Verlaufskurvenprozesse abzuwenden. Zum Interviewzeitpunkt lässt sich eine Annäherung an eine berufliche und private Verortung feststellen.

Für den Übergang vom Studium in den Beruf identifiziert Frau Winkler eine „Präphase“ vom Ethnologie- zum Medizinstudium (Z. 1766), die sich wurde eine lautsprachliche Transkription unter Einschluss paraverbaler und prosodischer Elemente verwendet. 
vor allem durch eine finanzielle Selbstständigkeit kennzeichnet. Dadurch, dass sie weiterhin Studentin ist und in einer Wohngemeinschaft lebt, erfolgt die Verantwortungsübernahme in dieser Phase noch in den Grenzen ihrer studentischen Rolle. Den ,eigentlichen, wirklichen“ Übergang vom Studium in den Beruf (Z. 1776 f.) stellt für sie der Übergang vom Medizinstudium zur klinischen Tätigkeit im Krankenhaus dar, den sie als großen „Bruch“ erlebt (Z. 1781). Dieser Übergang ist gekennzeichnet durch eine starke Veränderung ihres Tagesablaufs von studentischer Gestaltungsfreiheit hin zu einer starken Strukturierung durch die Vollzeitstelle und die Verantwortungsübernahme für Patientinnen in der Geburtshilfe und ihre Rollenübernahme als Ärztin. Frau Winkler konstatiert, dass Medizin als klassische Profession im Gegensatz zum Ethnologiestudium sehr stark auf das Berufsbild als Ärztin vorbereitet, die eigentliche Rollenübernahme jedoch einen schwierigen Bewältigungsprozess darstellt, der weitgehend ohne Unterstützung stattfindet und vor allem eine Frage des eigenen Zutrauens bedeutet. Hierzu führt Frau Winkler aus:

ich hatte wirklich ganz lange das Gefühl, ich spiele Ärztin also ich dachte immer, wenn ich so über den Flur gegangen bin in meinem Kittel und in meinen weißen Klamotten [...] mit dem Stethoskop in der Tasche, das ist auch ein bisschen wie Karneval gerade hier, und sobald ich im Kreißsaal war, hab ich [...] die Verantwortung übernommen und ich glaub ich bin auch eine gute Ärztin und hab viel positive Rückmeldung bekommen aber so dieses Selbstbild, ich arbeite hier gerade als Ärztin und ich muss unter Umständen in lebensbedrohlichen Situationen unter einer Geburt zum Beispiel halt richtig entscheiden [...] dass ich das wirklich konnte und auch gemacht hab, manchmal hab ich bis heute ein bisschen Schwierigkeiten das wirklich nachzuvollziehen (Z. 1820-1839).

\subsection{Die Mentoringbeziehung}

Frau Winklers Mentorin ist ca. 15 Jahre älter als sie und hat zwei Kinder im Alter von fünf und sieben Jahren. Sie ist Psychologin an einem wissenschaftlichen Institut, das sie selbst mit aufgebaut hat und das medizinische Themen in einem sozialwissenschaftlichen Sinne bearbeitet. Frau Winkler identifiziert deutliche Schwierigkeiten in Bezug auf die Mentoringbeziehung, da sie den Eindruck hat, dass ihre Mentorin wenig Interesse an der Beziehung und an ihrer Person zeigt. Sie hat das Gefühl zu ,stören“, weil die Treffen mit der Mentorin in deren Haus und in Anwesenheit der Kinder stattfinden. Sie stellt fest, dass kaum eine Weitergabe persönlicher Lebens- und Berufserfahrung erfolgt, sondern die Mentorin ihr eher einen inhaltlichen Einblick in Arbeitsprojekte bietet. Dennoch beschreibt sie eine starke Funktion der Mento- 
rin als Rollenvorbild sowie eine Verortungsfunktion und eine Entwicklung ihres Selbstbewusstseins.

Die Funktionen des Mentorings für Frau Winkler sollen im Folgenden strukturiert über die Mentoringbausteine der Mentoringbeziehung (1), der Peergroup (2) und des Workshopprogramms (3) dargestellt werden.

\subsection{Funktionen des Mentorings: (1) Mentorin}

Durch den Austausch mit der Mentorin lässt sich eine Stärkung von Frau Winklers Selbstbewusstsein feststellen. Ihr wird bewusst, was sie bislang erreicht hat. Sie reflektiert mit ihrer Mentorin die positive Rückmeldung aus ihrem Umfeld und stellt fest, dass sie sich selbst ernst nehmen kann und soll. Sie setzt ihre beruflichen Interessen, wie auch ihre Mentorin, gegen Widerstände durch (s. Abschnitt zur Vorbildfunktion). Frau Winkler evaluiert:

ich bin schon [...] sehr stolz darauf, dass ich das gemacht hab und dass ich das so geschafft hab und [...] deswegen war auch glaub ich das Mentoring so wichtig ((Luft)) an dem Punkt, weil ich (4) dieses Medizinstudium durchgezogen hab entgegen aller Fragen [...] also das war so [...] das, was mir im klinischen Alltag insbesondere am Anfang dann auch sehr geholfen hat, du hast das jetzt geschafft was soll denn eigentlich noch kommen (Z. 1912-1931).

Frau Winklers Mentorin übernimmt eine starke Vorbildfunktion, indem sie ihr vorlebt, dass sie ihre Interessen in der Verbindung von Medizin und Ethnologie auch gegen Widerstände durchsetzen und damit beruflich erfolgreich sein kann. Sie setzt dies in Beziehung zu ihren Fremdheits- und, wie sie es nennt, ,Alien“-Gefühlen:

es gibt andere Leute, du bist nicht der Alien (4) also wie eine Vorbildfunktion, an einem ganz bestimmten Punkt also nicht als Person an sich [...] sondern an diesem Punkt, sie hat gemacht was sie wollte [...] sie hat dieses Institut mit aufgebaut [...] auch gegen Widerstände [...] aber jetzt ist das halt ein total etabliertes Institut (Z. 1717-1720).

Ihre Entscheidung für ihre aktuelle berufliche Tätigkeit in der Wissenschaft beschreibt sie als direkte Konsequenz aus der Vorbildfunktion ihrer Mentorin:

so gesehen ist auch eigentlich das Projekt, was ich jetzt mache und dass ich mich doch noch mal für die Wissenschaft entschieden hab, jetzt auch schon eine Konsequenz [...] tatsächlich von der Mentorin weil sie das eben auch so gemacht hat [...] also man kann sich noch mal andere Sachen überlegen oder [...] wenn man etwas bestimmtes noch machen will, 
dann [...] würd ich das durchaus noch mal versuchen [...] denn es hat sonst ein bisschen was von nicht verwirklichten Träumen (Z. 2033-2048).

Ihre Mentorin zeigt ihr, dass sie ihren beruflichen Weg flexibel gestalten, sich entwickeln, verändern und ihre Wünsche verwirklichen kann. Sie vermittelt, dass Berufs- und Lebenswege nicht geradlinig sein müssen:

wenn es vielleicht nicht so der ganz geradlinige [...] Weg ist und du halt [...] keine 25 bist und anfängst Ärztin zu sein sondern halt schon 30 [...] also das hat meine Mentorin schon ziemlich stark ausgestrahlt (Z. 20042011).

Die Mentorin erfüllt zudem eine wichtige Verortungsfunktion im Rahmen der Suche von Frau Winkler nach sich selbst und ihrer Identität: Frau Winkler nimmt aufgrund ihres Wunsches nach Orientierung für ihre berufliche Zukunft am Mentoring teil und sucht nach einer Verbindung von Medizin und Ethnologie. Sie beschreibt dies folgendermaßen:

für mich war schwierig nicht so genau zu wissen, wo ich eigentlich hin will, ich hab immer die Sachen gemacht, die mich interessiert haben und wo meine Neugier hingegangen ist, aber dass es dafür auch einen Platz gibt in der Berufswelt, das hat mir eigentlich das Mentoringprogramm gezeigt und das hat mir wiederum auch die Mentorin gezeigt, es gibt hier einen Platz für dich (6) genau es gibt hier einen Platz für dich, interessant die Beziehung zu meiner Heimatlosigkeit (Z. 2153-2165).

Frau Winkler stellt demnach die Verortung durch die Mentorin, die ihr einen Platz in der Berufswelt zeigt, in Beziehung zu ihrer empfundenen Heimatlosigkeit.

\subsection{Funktionen des Mentorings: (2) Peergroup}

Die Peergroup ist für Frau Winkler von besonderer Relevanz, da sie ihr eine langjährige Beratung und Begleitung auf ihrem biographischen Weg ermöglicht. Nach dem offiziellen Ende des Mentoringprogramms führen die Mentees ihren Austausch als Stammtisch informell und eigeninitiativ weiter. Dabei erfüllt die Peergroup vor allem eine emotionale Unterstützungsfunktion, indem Frau Winkler durch den Vergleich mit den Mentee-Peers feststellt, dass diese ähnliche Unsicherheiten im Übergang bewältigen müssen und ebenfalls über, wie Frau Winkler formuliert, nicht ganz geradlinige Berufs- und Lebenswege verfügen. Die Peergroup ermöglicht in diesem Kontext Dialog und Austausch sowie die bereits erwähnte Beratungs- und Begleitungsfunktion. Frau Winkler beschreibt die Mentee-Peergroup, nicht zuletzt aufgrund der Schwierigkeiten in der Beziehung zur Mentorin, als für sie wichtigstes Element im Mentoring. 


\subsection{Funktionen des Mentorings: (3) Workshopprogramm}

Das Workshopprogramm dient für Frau Winkler der überfachlichen Kompetenzentwicklung. Sie hebt besonders einen Workshop zum Projektmanagement hervor, den sie als unabdingbar für ihre aktuelle berufliche Tätigkeit beschreibt. Frau Winkler evaluiert, dass die Projektmanagement-Ausbildung maßgeblich für die Bewilligung ihres Antrags bei der Forschungsförderungsgesellschaft war und diese ihr auch auf der handlungspraktischen Ebene die Durchführung ihres Projekts erst ermöglicht.

\subsection{Zusammenfassung Mentoring und Übergang}

Das Mentoring wirkt für Frau Winkler sehr stark in die Übergangsphase hinein, sowohl die Mentoringbeziehung als auch die fortwährende Begleitung durch die Mentee-Peers. Besonders relevant ist für sie der Baustein des PeerMentorings.

Das Mentoring vermittelt Frau Winkler in einer Gesamtbetrachtung über die verschiedenen Elemente hinweg, dass eine flexible, den eigenen Interessen folgende Gestaltung ihres Berufs- und Lebenswegs realisierbar ist. Dies erfordert Mut, sich gegen Widerstände zu behaupten, wobei eine biographische Anschlussfähigkeit möglich ist:

die [Mentorin] hat mir noch mal bestätigt, quasi aus der Position der bereits im Beruf Etablierten, dass das auch wirklich was ist, was beruflich wichtig ist, wenn man sich eben nicht unbedingt entscheiden möchte, was [...] man vielleicht für immer machen möchte, also so eine Flexibilität aber eben auch eine Konsequenz in dem eigenen Vorwärtsschreiten auf dem beruflichen Weg (Z. 2087-2094).

\section{Ausblick}

Die Falldarstellung veranschaulicht das Potenzial von Mentoring zur Begleitung von Transitionen: Mentoring eröffnet Möglichkeitsräume für Lern- und Entwicklungsprozesse durch die Initiierung biographischer Reflexivität und die Herstellung biographischer Anschlussfähigkeit im Übergang. Mentoring ist jedoch ein stark selektives Unterstützungsinstrument, das bereits eigenaktive Personen erreicht (vgl. Lorenzen 2014 ${ }^{8}$ ). In Bezug auf Prozessstrukturen

8 Lorenzen diskutiert v. a. den Übergang von der Schule in nachfolgende Systeme (Studium, Ausbildung), unter der Perspektive einer schulischen und sozialpädagogischen bzw. -arbeiterischen Unterstützung. Dennoch sind ihre Schlussfolgerungen vergleichbar zur Situation von Mentoring im Übergang Studium-Beruf unter einer erwachsenenpädagogischen Perspektive. 
lässt sich anhand des Materials fragen, ob Mentoring primär diejenigen erreicht, die ein biographisches Handlungsschema ausbilden. In Betrachtung der Funktionen von Mentoring und der Zielsetzungen zur Begleitung des Übergangs ist zudem zu überlegen, inwiefern Mentoring als ehrenamtliches Engagement und „Individuallösung“ eine Antwort auf bildungspolitische Zielsetzungen und strukturelle Probleme darstellen kann. So stellt Lorenzen fest, dass durch die Expansion von Beratung als Übergangsmanagement „gemeisterte aber auch gescheiterte Übergänge in erster Linie als individuelle Erfolge bzw. Versagen der Übergangsgestaltung [erscheinen] [...]“ (Lorenzen 2014, S. 140 f.). Damit besteht die „Gefahr, dass Mentoring als Beratung sozialpolitisch funktionalisiert wird und Probleme, negative Folgen und Risiken der Modernisierungsprozesse weiter individualisiert werden. Damit werden soziale Probleme nicht gelöst, sondern ihre strukturelle Existenz verschleiert" (Lorenzen 2014, S. 153). Diese Fragen sollen in die weitere Analyse einbezogen werden.

\section{Literatur}

Beck, U. (1986): Risikogesellschaft. Auf dem Weg in eine andere Moderne. Frankfurt a. M.: Suhrkamp.

Beck, U./Giddens, A./Lash, S. (1996): Reflexive Modernisierung. Eine Kontroverse. Frankfurt a. M.: Suhrkamp.

Berkels, B. (2014): Lernen im Rahmen von Mentoringprozessen - Potentiale und Grenzen eines Bildungsberatungsformats im Übergang vom Studium in den Beruf. In: Schlüter, A. (Hrsg.): Beratungsfälle - Fallanalysen für die Lern- und Bildungsberatung. Opladen: Barbara Budrich, S. 103-128.

Haasen, N. (2001): Mentoring. Persönliche Karriereförderung als Erfolgskonzept. München: Heyne.

Kurmeyer, C. (2012): Mentoring. Weibliche Professionalität im Aufbruch. Wiesbaden: VS Verlag für Sozialwissenschaften.

Lorenzen, J.-M. (2014): Aktiviert im Übergang? Bestimmung von Mentoring als Form der Beratung in aktivierungslogischem Zusammenhang. In: Lorenzen, J.M./Schmidt, L.-M./Zifonun, D. (Hrsg.): Grenzen und Lebenslauf. Beratung als Form des Managements biographischer Übergänge. Weinheim, Basel: Beltz Juventa, S. 140-156.

Oehme, A. (2007): Übergänge in Arbeit. Kompetenzentwicklung, Aneignung und Bewältigung in der entgrenzten Arbeitsgesellschaft. Baltmannsweiler: Schneider Verlag Hohengehren.

Pohl, A./Stauber, B./Walther, A. (2006): Zur Bedeutung informeller und partizipativer Lernprozesse für die Übergänge junger Erwachsener in die Arbeit. In: Tully, C. J. (Hrsg.): Lernen in flexibilisierten Welten. Wie sich das Lernen der Jugend verändert. Weinheim: Juventa, S. 183-199.

Schell-Kiehl, I. (2007): Mentoring: Lernen aus Erfahrung? Biographisches Lernen im Kontext gesellschaftlicher Transformationsprozesse. Bielefeld: Bertelsmann. 
Schlüter, A. (2010): Bildungsberatung. Eine Einführung für Studierende. Opladen: Barbara Budrich.

Schütze, F. (1983): Biographieforschung und narratives Interview. In: Neue Praxis, Jg. 13, H. 3, S. 283-293.

Sickendiek, U./Engel, F./Nestmann, F. (2008): Beratung. Eine Einführung in sozialpädagogische und psychosoziale Beratungsansätze. Weinheim, München: Juventa.

Welzer, H. (1990): Zwischen den Stühlen. Eine Längsschnittuntersuchung zum Übergangsprozeß von Hochschulabsolventen. Weinheim: Deutscher Studien Verlag.

Welzer, H. (1993): Transitionen. Zur Sozialpsychologie biographischer Wandlungsprozesse. Tübingen: edition diskord. 